\section{In Situ Probes for Measurement of Electrical Conductivity of Soilless Substrates: Effects of Temperature and Substrate Moisture Content}

\author{
Holly L. Scoggins \\ Department of Horticulture, 301-C Saunders Hall, Virginia Tech, Blacksburg, \\ VA 24061
}

\author{
Marc W. van Iersel \\ Department of Horticulture, 1111 Plant Science Building, University of Georgia, \\ Athens, GA 30602
}

Additional index words. electrical conductivity, fertilizer, soilless media, pour-through, 1:2 dilution, saturated media extract, time domain reflectometry, TDR

\begin{abstract}
Growing medium electrical conductivity (EC) is used in laboratory analysis and greenhouse production as a measure of the nutrient content of the growing medium. Fast, accurate ways to measure growing medium EC will make it easier to determine EC and maintain it within a suitable range for a particular crop. Several probes have been developed that can be inserted directly into the growing medium of container-grown crops for measurement of EC. We tested the sensitivity of four in situ EC probes (Field Scout, HI 76305, WET sensor, and SigmaProbe) at a range of temperatures, substrate volumetric water contents (VWC), and fertilizer concentrations. The HI 76305 probe was highly sensitive to temperature, while the WET sensor was temperature-sensitive at high ECs above its normal operating range. The probes responded differently to increasing VWC. The SigmaProbe and WET sensor measure the EC of the pore water specifically and show a decrease in EC with increasing water content, as the fertilizer ions in the pore water become more diluted as VWC increases. EC readings of the HI 76305 and Field Scout probes, which measure the EC of the bulk substrate (growing medium, water, and air combined) increased with increasing water content as the added water helps conduct the current of these meters. At a VWC above 35\%, there was little effect of VWC on EC readings of all probes. The EC measured with the various in situ probes differed slightly among the probes but was highly and positively correlated with all three of the standard solution extraction methods [pour-through, 1:2 dilution, and saturated media extract (SME)] over the range of fertilizer concentrations at a given temperature and VWC. These results make it possible to convert substrate $\mathrm{EC}$ guidelines that have been established for any of the three standard methods for use with the in situ probes, though our results indicate the substrate VWC must be above $35 \%$ for the interpretation to be valid. The in situ probes are a viable alternative for measurements of substrate $\mathrm{EC}$ and eliminate the step of substrate solution extraction, thus simplifying data collection.
\end{abstract}

For many floriculture and greenhouse crops, current substrate $\mathrm{pH}$ and electrical conductivity (EC) interpretation ranges are based on three substrate solution extraction methods: 1) 1 substrate : 2 water (by volume) suspension (1:2 dilution) (Sonneveld, 1990); 2) the saturated media extract (SME) method (Warncke, 1986); or 3) the pour-through extraction method (Cavins et al., 2004; Wright, 1986). The first two methods are generally used by analytical labs, while the pour-through method was developed as a quick and inexpensive test that can be performed in the greenhouse or nursery. Although the pour-through method is relatively quick, collection and measurement of many

Received for publication 29 June 2005. Accepted for publication 30 Oct. 2005. Our thanks to Fafard, Inc., Anderson, S.C. for providing the growing substrate. Mention of brands and products does not imply endorsement of this product by Virginia Tech or the University of Georgia.

${ }^{1}$ To whom reprint requests should be addressed; e-mail hollysco@vt.edu. samples is still time-consuming. Since many greenhouse growers produce a wide range of crops, which may have different nutrient requirements, a faster and simpler way to measure substrate EC and $\mathrm{pH}$ would be beneficial. In situ measurements of substrate EC eliminate the step of extracting the substrate solution, and speed up data collection. In recent years, several probes have been developed that can be inserted directly into the growing medium to get $\mathrm{EC}$ or $\mathrm{pH}$ measurements. However, interpretation of the EC readings from such probes is difficult, because crop guidelines generally have been developed based on measurements using the 1:2 dilution, SME, or pour-through method (Lang, 1996). Since different measurement techniques give different results, guidelines developed for one method cannot be used to make recommendations based on data collected with a different method. To ease interpretation of data collected with any of the in situ probes, it is important to determine how those data compare to measurements collected with the methods for which guidelines have been established. Although several in situ probes are currently available, there is little information on their accuracy.

Our objectives were to 1) compare results from in situ probes with currently used methods of EC salt measurement; 2) test the temperature sensitivity of the various in situ probes; and 3) determine the effect of the substrate moisture content on in situ probes. The goal of this research was to evaluate whether these EC probes are suitable for greenhouse use, and to provide data that will assist the development of guidelines for the interpretation of the data from these probes.

\section{Materials and Methods}

Probes. Four different in situ EC probes were tested: the HI 76305 (Hanna Instruments, Woonsocket, R.I.), Field Scout (Spectrum Technologies, Plainfield, Ill.), SigmaProbe (Delta-T Devices, Burwell, U.K.) and WET sensor (Delta-T Devices) (Table 1). The HI 76305 is an amperometric steel probe that can be inserted directly into the soil or substrate and is connected to a HI 993310 portable water conductivity and soil salinity meter. The probe measures the total conductivity of the soil, i.e., the combined conductivity of air, water, and soil particles or substrate components. The probe is factory-calibrated and calibration cannot be changed by the user. The probe does not correct EC measurements for substrate temperature. The manufacturer's information refers to soil salinity rather than electrical conductivity and the measurements are reported in units of $\mathrm{g} \cdot \mathrm{L}^{-1}$. Comparing EC to salinity can be inexact. Conversion factors from $\mathrm{EC}\left(\mathrm{dS} \cdot \mathrm{m}^{-1}\right)$ to salinity when measured in $\mathrm{mg} \cdot \mathrm{L}^{-1}$ range from $1 \mathrm{dS} \cdot \mathrm{m}^{-1}=640 \mathrm{mg} \cdot \mathrm{L}^{-1}$ to 1 $\mathrm{dS} \cdot \mathrm{m}^{-1}=700 \mathrm{mg} \cdot \mathrm{L}^{-1}$, depending on the fertilizer composition (Whipker and Cavins, 2000). Prior work with the HI 76305 by Pennisi and van Iersel (2002) found that a conversion factor of 1 $\mathrm{dS} \cdot \mathrm{m}^{-1}=0.155 \mathrm{~g} \cdot \mathrm{L}^{-1}$ gave results similar to EC measurements using the pour-through method. Since there seems to be a discrepancy in the conversion factor, data from the Hanna 76305 are given in the salinity units as measured and are not converted to EC units.

The Field Scout also measures total soil (or substrate) conductivity, but has automatic temperature compensation with a correction factor $(\beta)$ of $2 \%$ per ${ }^{\circ} \mathrm{C}$ and a reference temperature of $25{ }^{\circ} \mathrm{C}$. The probe can be user-calibrated using regular EC standards. Two different Field Scout meters were used in this study, and gave similar results, so data from the two probes were averaged.

The HI 76305 and Field Scout use a similar operating principle as standard solution EC probes; a voltage is applied to electrodes in the sensor (one pair of electrodes for the HI 76305, two pairs for the Field Scout) and the resulting current is measured. Using Ohm's law, the conductance of the soil or substrate can then be calculated. Guidelines for both the HI 76305 probe and Field Scout recommend that readings are taken in well-watered substrates. 


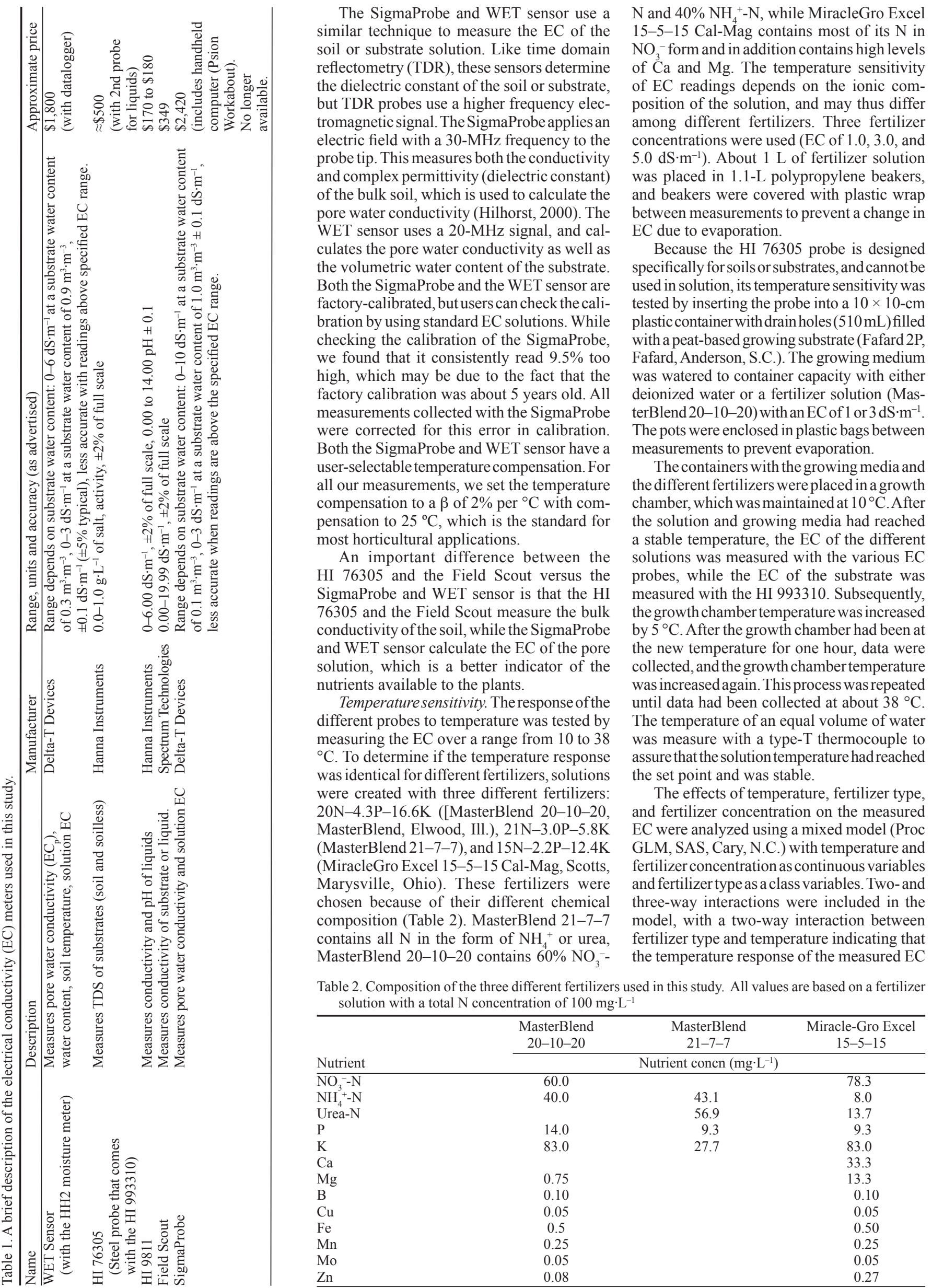


differed among fertilizers. This analysis was done separately for each probe. Data collected with HI 76305 probe were analyzed with a general linear model with fertilizer concentration as a class variable and temperature as a continuous variable.

Volumetric moisture content (VWC). To create ascending substrate moisture levels, $100,200,300$, and $400 \mathrm{~mL}$ deionized water was added to a $1100-\mathrm{mL}$ polypropylene beaker with $1 \mathrm{~L}$ of substrate (Fafard 3B, VWC in bag $=0.07)$, stirred thoroughly, and in $2 \mathrm{~h}$, stirred again and measured. A 0 treatment consisted of substrate directly from the bag, with no additional water. Substrate temperature at the time of testing was $22.6^{\circ} \mathrm{C}$. Substrate VWC was measured with the Delta-T HH2/WET. Sensor moisture meter and ranged from 0.03 to $0.55 \mathrm{~m}^{3} \cdot \mathrm{m}^{-3}$. The in situ probes were inserted into the substrate three times (each in a different location within the beaker) to serve as three subsamples. Measurements were taken randomly among the five moisture levels.

Fertilizer concentrations. A water-soluble fertilizer[15N-1.4P-12.4K(MiracleGro Excel 15-5-15 Cal-Mag)] was added to deionized (DI) water to achieve concentrations of 0.5 , $1.5,2.5,3.5,4.5$, and $5.5 \mathrm{dS} \cdot \mathrm{m}^{-1}$ and DI water $\left(0 \mathrm{dS} \cdot \mathrm{m}^{-1}\right)$. A solution concentration of 0.5 $\mathrm{dS} \cdot \mathrm{m}^{-1}$ from this fertilizer corresponds to about $75 \mathrm{mg} \cdot \mathrm{L}^{-1}$ of N. In total, $800 \mathrm{~mL}$ of these solutions was added to plastic bags containing $2 \mathrm{~L}$ of Fafard 3B (Fafard, Inc.), a soilless substrate containing peat moss, perlite, vermiculite, and processed pine bark. Bag contents were mixed manually for several minutes to ensure thorough dispersal, set aside for $1.5 \mathrm{~h}$, then remixed before testing. Substrate volumetric water content (VWC) percentage was $0.56,0.52$, $0.49,0.53,0.49,0.53$, and 0.52 , respectively, for the 0 to $5.5 \mathrm{dS} \cdot \mathrm{m}^{-1}$ treatments (each is a mean of 3 readings) as measured by the WET Sensor. Substrate temperature at the time of testing was $23.8^{\circ} \mathrm{C}$. Four in situ probes were used to measure substrate EC or salinity. The in situ probes were inserted into the substrate three times (each in a different location within the bag) at depths from 3 to $9 \mathrm{~cm}$ to serve as three samples. Probes and electrodes were rinsed with DI water between measurements. Measurements were taken randomly among the seven fertilizer treatments.

In addition to the direct measurements, substrate solution was extracted by three different methods. For the 1:2 dilution, $430 \mathrm{~mL}$ of DI water was added to $215 \mathrm{~mL}$ substrate, stirred, and allowed to calibrate for $30 \mathrm{~min}$. For the pour-through extraction method, 10.6- $\mathrm{cm}$ containers $(575 \mathrm{~mL}$ volume) were filled with the prepared substrate, allowed to settle for 1 $\mathrm{h}$, and then $50 \mathrm{~mL}$ DI water was poured over the substrate surface. Leachate was collected in vinyl saucers, transferred to beakers, and measured. For the SME procedure, $450 \mathrm{~mL}$ of substrate was saturated to glistening with DI water and allowed to equilibrate for 30 min, after which the slurry was vacuum-filtered through a Büchner funnel lined with Whatman's \#1 filter paper. Both EC and pH of the extracted solution were measured with a Hanna 9811 EC and pH meter.

\section{Results and Discussion}

Temperature sensitivity. Many conductivity meters are described as automatically temperature compensating by the manufacturer most commonly with a $\beta$ of $2 \%$ per ${ }^{\circ} \mathrm{C}$, although it is used-selectable for some probes. However, the temperature sensitivity of the EC depends on the ionic composition of the solution, and a $\beta$ of $2 \%$ per ${ }^{\circ} \mathrm{C}$ may not always be appropriate. Also, it is possible that some of the electronics in the probe are temperature sensitive, causing an additional measurement error. Our results showed the in situ probes varied in their responses to changing temperatures (Fig. 1). The HI 76305 probe was extremely temperature-sensitive, with readings increasing by over $100 \%$ in a growing medium with DI water. Measurements increased from $<4 \mathrm{~g} \cdot \mathrm{L}^{-1}$ at $10^{\circ} \mathrm{C}$ to $9 \mathrm{~g} \cdot \mathrm{L}^{-1}$ at $38^{\circ} \mathrm{C}$. There was a similar absolute increase in the measured salinity levels in growing media saturated with solutions with an EC of 1 or 3 $\mathrm{dS} \cdot \mathrm{m}^{-1}$. The Hanna 9811 solution probe and the SigmaProbe showed the least sensitivity
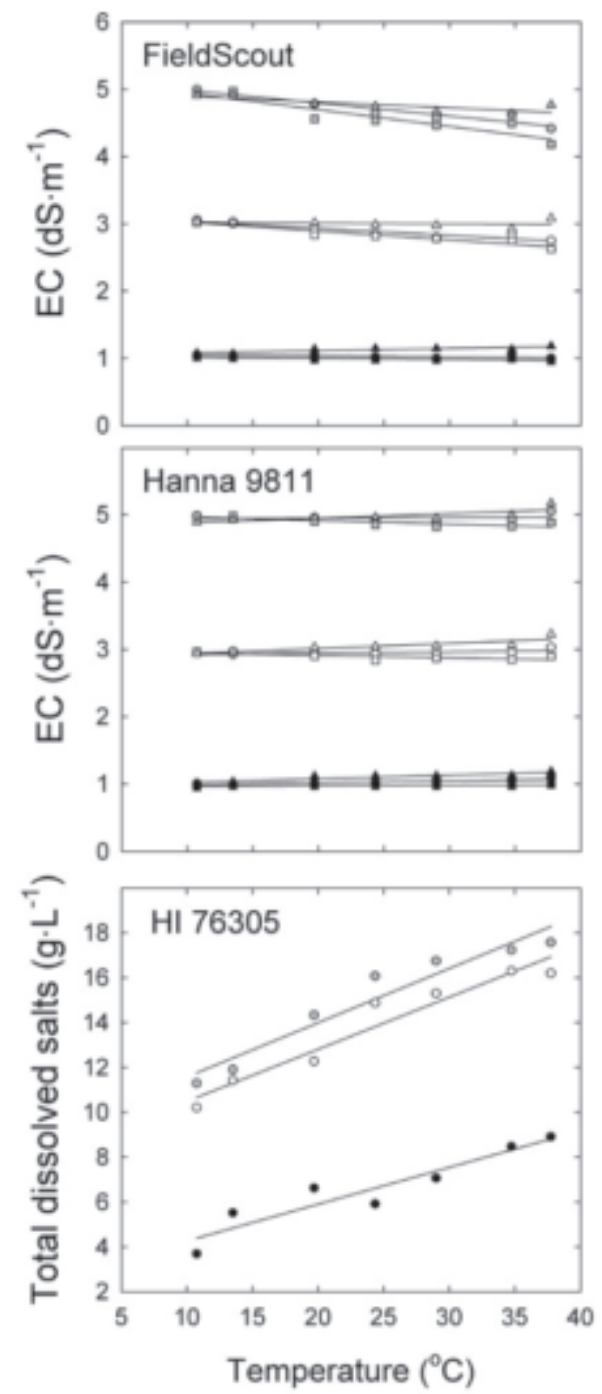

Fig. 1. Temperature sensitivity of various electrical conductivity (EC) or salinity probes. The EC of three different concentrations of three different fertilizers was measured with the Field Scout, SigmaProbe, Hanna 9811, and WET probe at temperatures ranging from 10 to $38^{\circ} \mathrm{C}$. Because the $\mathrm{HI} 76305$ probe can only be used to measure salinity in soils or substrates, it was tested in a peat-based growing medium saturated with 20-10-20 fertilizer solution with an EC of 0 (solid circle), 1 (open circle), or 3 (shaded circle) $\mathrm{dS} \cdot \mathrm{m}^{-1}$. to temperature, independent of the EC of the solution. The Field Scout tended to slightly overcompensate for temperature, especially at higher EC levels ( 3 and $5 \mathrm{dS} \cdot \mathrm{m}^{-1}$ ), i.e., the measured EC decreased with increasing temperature. The WET sensor performed well in solution with an EC of $1 \mathrm{dS} \cdot \mathrm{m}^{-1}$, slightly undercompensated in a solution with an EC of $3 \mathrm{dS} \cdot \mathrm{m}^{-1}$, and greatly undercompensated in a solution with an EC of $5 \mathrm{dS} \cdot \mathrm{m}^{-1}$. However, an EC of $5 \mathrm{dS} \cdot \mathrm{m}^{-1}$ in solution is beyond the normal operating range for this sensor, and the manufacturer does not make any claims for accuracy in such solutions.

The temperature response of the Field Scout, Hanna 9811 solution probe, and SigmaProbe was affected by the type of fertilizer $(P<0.001)$. For these probes, the temperaturecompensated EC of 21-7-7 solutions increased more (or decreased less, depending on the probe) with increasing temperature, while the temperature-compensated EC of $15-5-15 \mathrm{Ca}-$ Mag solutions decreased the most (or increased the least) with increasing temperature. These

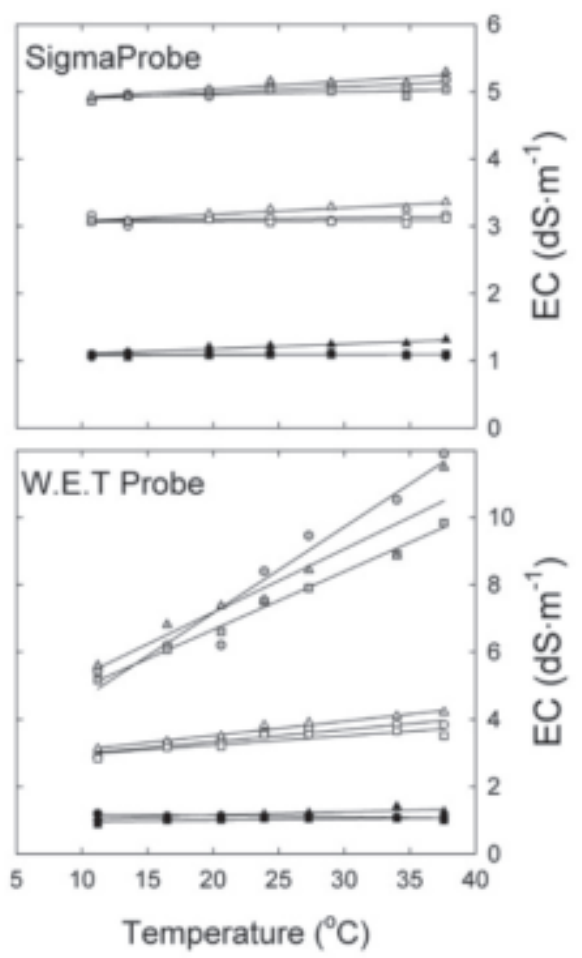

- 20-10-20, $E C=1 \mathrm{dS} \mathrm{m}^{-1}$

- 15-5-15, EC $=1 \mathrm{dS} \mathrm{m}^{-1}$

- 21-7-7, EC $=1 \mathrm{dS} \mathrm{m}^{-1}$

$20-10-20, E C=3 d S m^{-1}$

15-5-15, EC $=3 \mathrm{dS} \mathrm{m}^{-1}$

21-7.7. EC $=3 d \mathrm{dS} \mathrm{m}^{-1}$

20-10-20, EC $=5 \mathrm{dS} \mathrm{m}^{-1}$

- 15-5-15, EC $=5 d S \mathrm{~m}^{-1}$

- 21-7-7, $E C=5 d S m^{-1}$ 


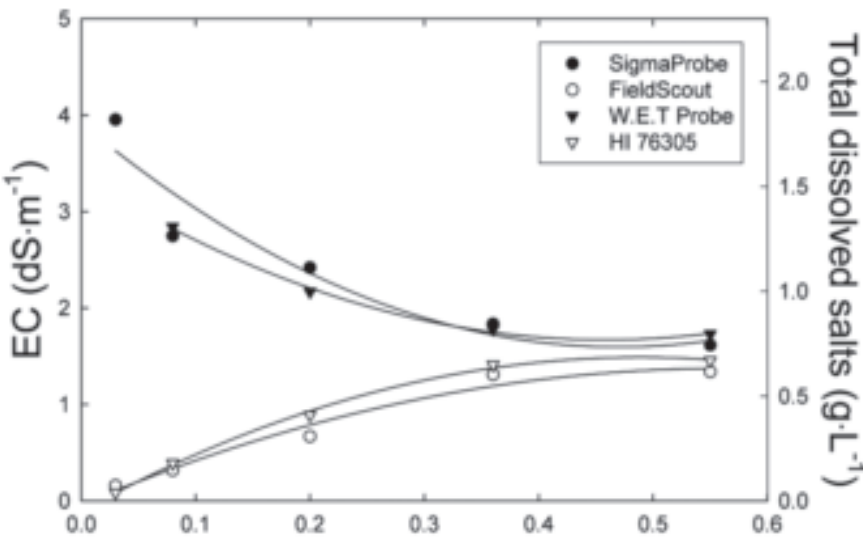

Substrate volumetric water content $\left(\mathrm{m}^{3} \cdot \mathrm{m}^{-3}\right)$ drought stress when the VWC is 0.10 to $0.15 \mathrm{~m}^{3} \cdot \mathrm{m}^{-3}$ (Nemali and van Iersel, 2004). The Hanna and Field Scout in situ probes measure the EC of the bulk substrate (substrate components, substrate solution, and air spaces), and their EC readings increase with increasing water because the added water displaces air.Air, even at close to $100 \%$ relative humidity, is a very poor conductor

Fig. 2. The effect of the volumetric water content (VWC) of a peat-based growing medium on its electrical conductivity (EC) or salinity, as measured by different in situ probes. Different water contents were created by adding different amounts of deionized water to the substrate, so the total amount of salts in the substrate was the same at all moisture contents. SigmaProbe: $\mathrm{EC}=3.92-9.88 \times \mathrm{VWC}+10.5 \times \mathrm{VWC}^{2}, r^{2}=$ 0.91; Field Scout: $\mathrm{EC}=-0.04+5.02 \times \mathrm{VWC}$ $-4.46 \times \mathrm{VWC}^{2}, r^{2}=0.97$; WET probe: $\mathrm{EC}=$ $3.36-7.37 \times \mathrm{VWC}+8.04 \times \mathrm{VWC}^{2}, r^{2}=0.99$; $\mathrm{HI}$ 76305: $\mathrm{EC}=-0.05+3.00 \times \mathrm{VWC}-3.06 \times$ $\mathrm{VWC}^{2}, r^{2}=0.99$.

differences among the temperature responses in different fertilizer solutions presumably are related to the different ionic compositions of the fertilizer solutions. For example, temperature affects the activity of monovalent and divalent ions differently (Voipio et al, 1994), which may explain why the temperature sensitivity of the EC of the MiracleGro Excel 15-5-15 Cal-Mag fertilizer (with relatively high concentrations of the divalent $\mathrm{Mg}^{2+}$ and $\mathrm{Ca}^{2+}$ ) differed from the other two fertilizers. However, there does not appear to be enough detailed information about temperature effects on the EC of individual ions or fertilizer salts to predict how the EC of various fertilizers may be affected by temperature.

The interaction between temperature and fertilizer EC was not significant for the WET sensor, possibly because this probe was used outside of its normal operating range. Data collected with the WET sensor were noisier $\left(R^{2}\right.$ for the mixed model $=0.958)$ than data collected with the Field Scout, Hanna 9811 solution probe, and SigmaProbe $\left(R^{2}>0.998\right)$.

Volumetric moisture content (VWC). All four EC probes responded quadratically to increasing VWC, and results were significant (Fig. 2). The kind of response differed dramatically depending on the type of probe. The SigmaProbe and WET sensor measure the EC of the pore water specifically and show a decrease in EC with increasing water, since the fertilizer ions in the pore water become more diluted as more water is added to the substrate. The WET sensor was unable to measure the EC of the pore water when the VWC was $0.03 \mathrm{~m}^{3} \cdot \mathrm{m}^{-3}$, and is recommended for use in substrates with a VWC of at least $0.13 \mathrm{~m}^{3} \cdot \mathrm{m}^{-3}$. This is not a practical limitation for use in soilless substrates, because plant growth generally is inhibited by of electricity, and a decrease in air space and an increase in water content will increase the conductivity of the bulk substrate. Also, additional nutrients may come into solution from the cation exchange sites of the substrate components as more water is added to the substrate and the solution concentration decreases.

There was little change in the measured EC for any of the probes as the VWC increased from 0.36 to $0.55 \mathrm{~m}^{3} \cdot \mathrm{m}^{-3}$. In addition, the differences in EC readings among the three EC probes decreased with increasing VWC. These results make it clear that it is important to always measure the EC of the substrate at a similar moisture level (at least 0.36 $\mathrm{m}^{3} \cdot \mathrm{m}^{-3}$ ) to assure that the results are comparable. For practical purposes, it would be simplest to measure the EC of the substrate at a set time (e.g., 30 min) after watering the substrate to container capacity. Substrates used in this study hada high water-holding capacity. Other soilless substrates, especially with a high proportion of pine bark, may not be able to hold as much water, and the response to changing VWC may be different for such substrates.

Fertilizer concentrations. As expected, there were systematic differences in the measured EC among the three laboratory methods (Fig. 3). Pourments consistently had a higher EC than through measure-
SME measurements, which in turn resulted in higher EC values than the 1:2 dilution method. This is not surprising, since the pour-through method is based on the concept that the water that is poured on top of the substrate and displaces the pore water out of the bottom of the container. Thus the pore water supposedly is not diluted in this method. With the SME method, water is added to the substrate until it glistens. This results in a dilution of the nutrients in the substrate, and therefore would be expected to lower the EC. With the 1:2 dilution method, even more water is mixed with the substrate, and the nutrients are thus diluted even more than with the SME method, resulting in a lower EC.

The EC measured with the various in situ

Fig. 3. Correlations between the electrical conductivity (EC) measured by various laboratory methods (pour-through, top; saturated media extract (SME), middle; and 1:2 dilution method, bottom) and the EC measured by four different in situ probes. Different EC levels were created by adding the same volume, but different concentrations, of MiracleGro Excel 15-5-15 Cal-Mag water-soluble fertilizer to a peat-based substrate. Regression results are shown in Table 3.
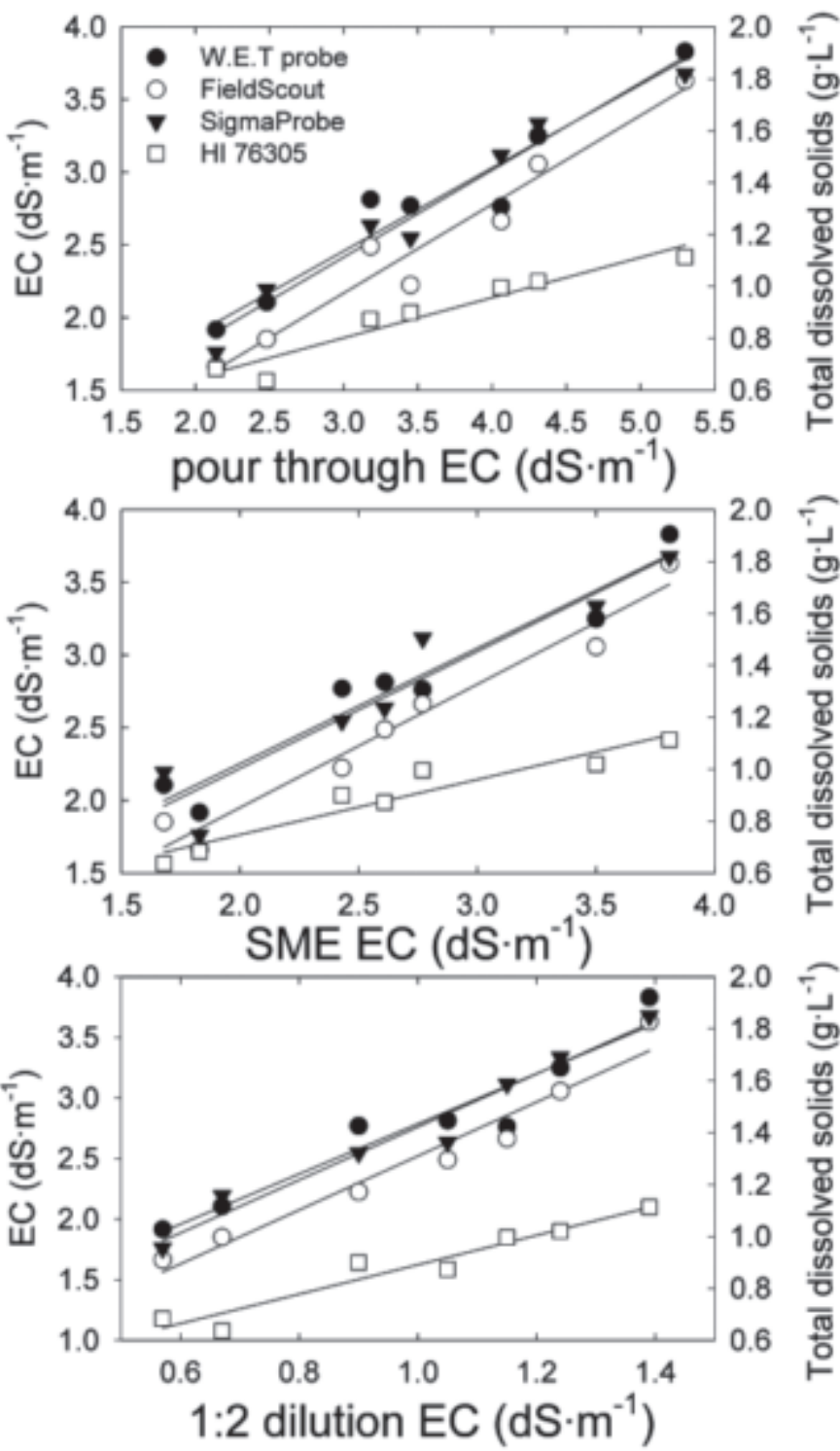
Table 3. Regression coefficients (estimate \pm standard error) of electrical conductivity (EC) measurements from in situ probes versus standard laboratory methods. To convert values from a standard laboratory method $(\mathrm{y})$ to an in situ probe measurements (x) use: $x=$ intercept + slope $\times \mathrm{y}$.

\begin{tabular}{llccc}
\hline In situ probe & Laboratory method & Intercept & Slope & $r^{2}$ \\
\hline WET probe & Pour-through & $0.74 \pm 0.25$ & $0.573 \pm 0.068$ & 0.93 \\
& $1: 2$ dilution & $0.71 \pm 0.29$ & $2.07 \pm 0.28$ & 0.92 \\
& Saturated media extract (SME) & $0.66 \pm 0.25$ & $0.795 \pm 0.091$ & 0.94 \\
SigmaProbe & Pour-through & $0.62 \pm 0.19$ & $0.599 \pm 0.051$ & 0.96 \\
& $1: 2$ dilution & $0.57 \pm 0.19$ & $2.19 \pm 0.18$ & 0.97 \\
& SME & $0.61 \pm 0.31$ & $0.805 \pm 0.115$ & 0.91 \\
Field Scout & Pour-through & $0.34 \pm 0.22$ & $0.611 \pm 0.059$ & 0.95 \\
& 1:2 dilution & $0.29 \pm 0.23$ & $2.23 \pm 0.22$ & 0.95 \\
HI 76305 & SME & $0.25 \pm 0.21$ & $0.849 \pm 0.077$ & 0.96 \\
& Pour-through & $0.34 \pm 0.07$ & $0.155 \pm 0.019$ & 0.93 \\
& $1: 2$ dilution & $0.33 \pm 0.07$ & $0.566 \pm 0.069$ & 0.93 \\
& SME & $0.32 \pm 0.08$ & $0.213 \pm 0.029$ & 0.91 \\
\hline
\end{tabular}

probes was highly and positively correlated with all three of the laboratory methods $(0.91<$ $r^{2}<0.97$; Fig. 3 , Table 3 ). This indicates that is possible to convert substrate EC guidelines that have been established for any of the laboratory methods to guidelines that can be used with the in situ probes. Thus, it is not necessary to develop new substrate EC guidelines for use with these in situ probes. However, it is important to realize that the regression analyses in Table 3 are valid only for well-watered substrates, since the measurements of all probes are sensitive to changes in the VWC of the substrate, if the VWC is below about $0.35 \mathrm{~m}^{3} \cdot \mathrm{m}^{-3}$. Thus, a thorough irrigation before use of the in situ probes is essential to get reliable comparisons with laboratory methods. In addition, data from the HI 76305 probe can only be compared to laboratory methods if the substrate temperature is close to $24{ }^{\circ} \mathrm{C}$, since this probe is highly sensitive to temperature.

The EC measured with the WET sensor, SigmaProbe, and Field Scout was consistently lower than the pour-through EC. The WET sensor, SigmaProbe, and pour-through differences were surprising since all determine the EC of the pore water. Further, measurements with the WET sensor and SigmaProbe were closer to those of the SME method than those of the Pour-through method. The WET sensor and SigmaProbe gave slightly higher EC values than the SME method at low substrate EC $\left(<2.5 \mathrm{dS} \cdot \mathrm{m}^{-1}\right)$, and were very similar to the SME method at high EC $\left(>3 \mathrm{dS} \cdot \mathrm{m}^{-1}\right)$. Conversely, the Field Scout resulted in similar values to the SME method at low substrate EC, and slightly lower values at high EC (Fig. 3 ). The WET sensor, SigmaProbe, and Field Scout all consistently resulted in higher EC readings than the 1:2 dilution method. Because of the different units, comparisons of the HI 76305 with standard laboratory methods are not possible.

Pore waterEC measurements from the WET sensor and SigmaProbe at different substrate EC levels were very similar. This is not surprising, since these two probes measure substrate EC based on the same principle, i.e., pore water $\mathrm{EC}$ is determined from the conductivity and complex permittivity of the bulk substrate(Hilhorst, 2000). The Field Scout measurements consistently were about $0.25 \mathrm{dS} \cdot \mathrm{m}^{-1}$ lower than those of the WET sensor or SigmaProbe. The lower measurements from the Field Scout likely are caused by the fact that this meter measures the EC of the bulk substrate, i.e., a combination of the air, water and substrate components in the container. Since both air and substrate components have a very low EC, this results in a lower EC than when the EC of just the pore water is measured (i.e., the WET sensor and SigmaProbe). However, the response of the Field Scout to increasing substrate EC was strikingly similar to those of the WET sensor and SigmaProbe, i.e., the slopes of the regression lines were similar (Fig. 3, Table 3). The HI 76305 also was very responsive to changes in substrate EC, but because of its unconventional units, it is difficult to compare its response to that of the other in situ probes.

Since EC (or salinity) measurements with all four in situ probes were very responsive to changes in substrate EC, all of these probes can potentially be used to monitor substrate EC in greenhouses or nurseries. However, use of the HI 76305 probe should probably be limited to conditions were temperature fluctuations are minimal.

Substrate samples in this experiment were purposely mixed before measurement to avoid stratification, but a caveat when using in situ probes may be where in the container the measurement is taken. Electrical conductivity varies depending on the depth of measurement and this effect is magnified by subirrigation (Argo and Biernbaum, 1995). Inserting the probe to a consistent substrate depth may help alleviate variation.

In summary, all four in situ EC probes were highly responsive to substrate $\mathrm{EC}$, as indicated by the strong correlation of the measurements of these probes to EC measurements with standard techniques. This strong correlation makes it possible to convert guidelines developed with standard techniques to recommendations that can be used to interpret data from the in situ probes. Unfortunately, the HI 76305 is highly temperature sensitive, which may limit its ap- plications to conditions where the temperature is relatively steady. The WET sensor becomes temperature sensitive at high EC, outside of the manufacturer-recommended operating range. Although the temperature correction on this meter is user-adjustable, we were not able to find a temperature correction that worked in all EC ranges (unpublished results). The standard temperature correction (to $25{ }^{\circ} \mathrm{C}$ with a $\beta$ of $2 \%$ per ${ }^{\circ} \mathrm{C}$ ) worked well for the Field Scout and SigmaProbe. The temperature sensitivity of all four probes depended on the kind of fertilizer that was used, but this fertilizer effect was probably not enough to be of practical importance.

Since measurements of all in situ EC probes were greatly affected by substrate VWC, it is important to standardize the substrate VWC for these measurements. Since the effect of VWC is small at high VWC, we suggest that measurements be taken in well-watered substrates, with a VWC of at least $0.35 \mathrm{~m}^{3} \cdot \mathrm{m}^{-3}$. For practical applications, testing the substrate within one hour of watering to container capacity should yield satisfactory results.

\section{Literature Cited}

Argo, W.R. and J.A. Biernbaum. 1995. The effect of irrigation method, water-soluble fertilization, preplant nutrient charge and surface evaporation on early vegetative and root growth of poinsettia. J. Amer. Soc. Hort. Sci. 120:163-169.

Cavins, J. T., B.E. Whipker, and W.C. Fonteno. 2004. Establishment of calibration curves for comparing pour-through and saturated media extract nutrient values. HortScience 39:1635-1639.

Hilhorst, M.A. 2000. A pore water conductivity sensor. Soil Sci. Soc. Amer. J. 64:1922-1925.

Lang, H.J. 1996. Growing media testing and interpretation, p. 123-139. In:D.W. Reed(ed.). Water, media, and nutrition for greenhouse crops, Ball Publ., Batavia, Ill.

Nemali, K.S. and M.W. van Iersel. 2004. Moisture stress for growth control: Physiological responses of salvia (Salvia splendens) and vinca (Catharanthus roseus). Proc. S. Nursery Assn. Res. Conf. 49:618-622.

Pennisi, B.V. and M. van Iersel. 2002. Three ways to measure medium EC. GMPro 22(1):46-48, 50.

Sonneveld, C. 1990. Estimating quantities of watersoluble nutrients in soils using a specific $1: 2$ by volume extract. Commun. Soil Sci. Plant Anal. 21:1257-1265.

Voipio, J., M. Pasternack, and K. MacLeod. 1994. Ion-sensitive microelectrodes, p. 275-316. In: D.C. Ogden (ed.). Microelectrode techniques: The Plymouth workshop handbook. 2nd ed. Company of Biologists, Cambridge, U.K.

Warncke, D.D. 1986. Analyzing growth media by saturation extraction procedure. HortScience $21: 223-225$.

Whipker, B.E. and T.J. Cavins. 2000. Electrical conductivity (EC): Units and conversions. Florex.002.http://www.ces.ncsu.edu/depts/hort/ floriculture/Florex/EC\%20Conversion.pdf.

Wright, R.D. 1986. The pour-through nutrient extraction procedure. HortScience 21:227-229. 